\title{
SYZYGY PAIRS IN A MONOMIAL ALGEBRA
}

\author{
KIYOSHI IGUSA AND DAN ZACHARIA
}

(Communicated by Donald S. Passman)

\begin{abstract}
In this paper we construct the set of "syzygy pairs" of a finitedimensional monomial algebra and use it to prove the following theorem.
\end{abstract}

Theorem A. (Finitistic global dimension theorem) Let $\Lambda$ be a monomial (zero relations ) algebra over a field $k$. Let $M$ be a $\Lambda$-module with finite injective dimension. Then

$$
\text { inj } \operatorname{dim}_{\Lambda} M \leq \operatorname{dim}_{k} \operatorname{rad} \Lambda
$$

This is an improvement on [GKK] who were the first to show that monomial algebras satisfy the finitistic global dimension conjecture.

Conjecture. (A. Rosenberg and D. Zelinsky) Let $\Lambda$ be a finite-dimensional algebra over a field $k$. Then the injective dimension of the $\Lambda$-modules with finite injective dimension is bounded.

One immediate consequence of Theorem $\mathrm{A}$ is the following:

Corollary B. Let $\Lambda$ be a monomial algebra with finite global dimension. Then

$$
\text { gldim } \Lambda \leq \operatorname{dim}_{k} \operatorname{rad} \Lambda \text {. }
$$

The following example shows that, in some sense this is the best possible bound.

Example. Let $\Lambda$ be the quotient of the $(n+1) \times(n+1)$ lower triangular matrix ring with entries in $k$ by the square of its radical. In other words $\Lambda$ is given by the quiver $A_{n+1}$ :

$$
\bullet v_{v_{o}} \rightarrow \bullet_{v_{1}} \rightarrow \cdots \rightarrow \bullet \bullet_{v_{n}}
$$

with the relations: any path of length $\geq 2$ is zero. Then gldim $\Lambda=n=$ $\operatorname{dim}_{k} \operatorname{rad} \Lambda$.

We briefly recall the definition of a finite-dimensional monomial algebra. Let $Q$ be a finite directed graph. Then $k Q$, the path algebra of $Q$, is the algebra spanned as a vector space by all the directed paths in $Q$. The multiplication of two paths is their composition, or zero if they are not composable. A monomial

Received by the editors December 5, 1988 and, in revised form, January 18, 1989.

1980 Mathematics Subject Classification (1985 Revision). Primary 16A46, 16A64.

The first author was partially supported by the National Science Foundation. 
algebra is a quotient of $k Q$ by an ideal generated by paths of length at least two. We refer the reader to [GHZ] for more details.

Throughout this paper, $\Lambda$ will denote a finite-dimensional monomial algebra. The modules are left $\Lambda$ modules.

\section{THE MAIN THEOREM}

The injective dimension of $M$ is the largest integer $n$ such that $\operatorname{Ext}_{\Lambda}^{n}(S, M) \neq 0$ for some simple $\Lambda$-module $S$. The problem is now very easy since the minimal projective resolution of a simple module has a very nice form. There are only a finite number of indecomposable modules which occur as summands of syzygies of simple modules. (We call these modules $K_{\gamma}$.) Therefore after a finite number of steps, the projective resolution of $S$ starts repeating and this leads to a bound on the dimension of $\operatorname{Ext}_{\Lambda}^{*}(S, M)$.

We now construct the modules $K_{\gamma}$ which occur as summands of syzygies of simple modules. If $Q$ is the quiver of $\Lambda$, let $S_{v}$ denote the simple module at the vertex $v$, and let $P_{v}$ be its projective cover.

Let $\gamma$ be a path in the quiver of $\Lambda$ of length $\geq 1$ so that $\gamma$ contains no zero relations (i.e. $\gamma \neq 0$ in $\Lambda$ ). Suppose that $\gamma$ starts at $v$ and ends at $w$. Then $\gamma$ gives a homomorphism of $\Lambda$ modules $\gamma^{*}: P_{w} \rightarrow P_{v}$ which is nonzero at $w$. Let $K_{\gamma} \subseteq P_{v}$ be the image of this homomorphism. Then the pair $\left(P_{v}, K_{\gamma}\right)$ will be called a syzygy pair for $\Lambda$. A homomorphism of syzygy pairs is defined in the obvious way.

Lemma 1.1. (a) Syzygy pairs corresponding to different paths are not isomorphic.

(b) The number of isomorphism classes of syzygy pairs equals the dimension of $\operatorname{rad} \Lambda$ as a k-vector space.

Proof. (b) follows from (a) because the nonzero paths of length $\geq 1$ form a basis of the radical of $\Lambda$. To prove (a) suppose that $\left(P_{u}, K_{\alpha}\right) \simeq\left(P_{v}, K_{\beta}\right)$. Then $u=v$ and $\alpha, \beta$ must be paths from $v$ to the same vertex, say $w$. The isomorphism $K_{\alpha} \simeq K_{\beta}$ lifts to an isomorphism $P_{w} \simeq P_{w}$ making the following diagram commute:

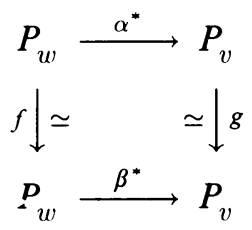

But then

$$
\begin{aligned}
g \alpha^{*}\left(e_{w}\right) & =a \alpha+\sum a^{\prime} \text { (longer paths) }=\beta^{*} f\left(e_{w}\right) \\
& =b \beta+\sum b^{\prime} \text { (longer paths) where } a, b \in k^{*} \text { and } a^{\prime}, b^{\prime} \in k .
\end{aligned}
$$

This implies that $\alpha=\beta$.

The following was essentially proved in [GHZ]. 
Lemma 1.2. Let $\gamma: v \rightarrow w$ be a path of length $\geq 1$ in the quiver of $\Lambda$. Then the kernel of the induced map $\gamma^{*}: P_{w} \rightarrow P_{v}$ is the direct sum of the submodules $K_{\gamma_{i}}$ of $P_{w}$ where $\gamma_{i}$ ranges over all paths satisfying the following conditions:

(1) $\gamma_{i}: w \rightarrow u_{i}$ is a path starting at $w$.

(2) $\gamma \gamma_{i}: v \rightarrow u_{i}$ contains exactly one (minimal) zero relation ending at $u_{i}$. Proof. Let $\widetilde{Q}$ be the universal covering of the quiver $Q$ of $\Lambda$. Then $\widetilde{Q}$ is an infinite tree (or disjoint union of trees) and all the zero relations in $Q$ lift to $\widetilde{Q}$. Let $\tilde{\gamma}: \tilde{v} \rightarrow \widetilde{w}$ be a lifting of $\gamma$. Then we have an induced map $\tilde{\gamma}^{*}: P_{\tilde{u}} \rightarrow P_{\tilde{v}}$ and the kernel of $\gamma^{*}$ is the push down of the kernel of $\tilde{\gamma}^{*}$. Thus we are reduced to finding the summands of $\operatorname{Ker} \tilde{\gamma}^{*} \subseteq P_{\tilde{w}}$. The result follows if we use the following well-known fact concerning representations of trees with relations: if $X$ is a representation with simple top, then $Y$ is a direct sum of representations with simple top, where $0 \rightarrow Y \rightarrow P \rightarrow X \rightarrow 0$ is exact and $P$ is the projective cover representation of $X$. It is now clear that $\operatorname{Ker} \gamma^{*}=\amalg K_{\gamma_{i}}$, where $\gamma_{i}$ satisfy conditions (1) and (2).

Definition 1.3. If $\left(P_{v}, K_{\gamma}\right)$ is a syzygy pair, let $\Omega^{1}\left(P_{v}, K_{\gamma}\right)$ denote the set of all syzygy pairs $\left(P_{w}, K_{\gamma_{i}}\right)$ where the $\gamma_{i}$ are as given in Lemma 1.2. Then, for each pair $\left(P_{w}, K_{\gamma_{i}}\right)$ of $\Omega^{1}\left(P_{v}, K_{\gamma}\right)$ we construct $\Omega^{1}\left(P_{w}, K_{\gamma_{i}}\right)$ by applying 1.2 and we denote by $\Omega^{2}\left(P_{v}, K_{\gamma}\right)$ the union of all $\Omega^{1}\left(P_{w}, K_{\gamma_{i}}\right)$. Inductively, let $\Omega^{n}\left(P_{v}, K_{\gamma}\right)$ be the union of the sets $\Omega^{n-1}\left(P_{w}, K_{\gamma_{i}}\right)$ for $n \geq 2$.

Lemma 1.4. Let $(P, K) \in \Omega^{n}\left(P^{\prime}, K^{\prime}\right)$ and let $\cdots \rightarrow P_{n}\left(K^{\prime}\right) \stackrel{d_{n}}{\rightarrow} P_{n-1}\left(K^{\prime}\right) \rightarrow$ $\cdots \rightarrow P_{0}\left(K^{\prime}\right) \rightarrow K^{\prime} \rightarrow 0$ be a minimal projective resolution of $K^{\prime}$. Then there is a summand $A$ of $P_{n-1}\left(K^{\prime}\right)$ and a summand $B$ of $d_{n} P_{n}\left(K^{\prime}\right)$ such that $B \subseteq A$ and $(A, B) \simeq(P, K)$.

Proof. This follows from Lemma 1.2 by induction on $n$.

Definition 1.5. We say that a syzygy pair $(P, K)$ is periodic if $(P, K)$ is isomorphic to an element of $\Omega^{n}(P, K)$ for some $n \geq 1$. The smallest such $n$ is called the period of $(P, K)$. We say that $(P, K)$ is virtually periodic if $(P, K)$ is isomorphic to an element of $\Omega^{n}\left(P^{\prime}, K^{\prime}\right)$ for some periodic pair $\left(P^{\prime}, K^{\prime}\right)$ and some $n \geq 1$.

Note that every periodic syzygy is virtually periodic.

Lemma 1.6. Suppose that $(P, K)$ is a virtually periodic syzygy pair and $M$ is a $\Lambda$-module with finite injective dimension. Then any homomorphism $K \rightarrow M$ extends to a homomorphism on $P$.

Proof. Let $\left(P^{\prime}, K^{\prime}\right)$ be a periodic syzygy pair of period $p$ so that $(P, K) \in$ $\Omega^{n}\left(P^{\prime}, K^{\prime}\right)$. Then $(P, K) \in \Omega^{n+p m}\left(P^{\prime}, K^{\prime}\right)$ for all $m \geq 1$. But $\operatorname{Ext}_{\Lambda}^{n+p m}\left(K^{\prime}, M\right)$ $=0$ for sufficiently large $m$, so $\operatorname{Hom}_{\Lambda}(P, M)$ must map onto $\operatorname{Hom}_{\Lambda}(K, M)$ by Lemma 1.4 . 
Lemma 1.7. Let $\left(P_{0}, K_{0}\right)$ be any syzygy pair, and let $n \geq \operatorname{dim}_{k} \operatorname{rad} \Lambda$. Then every element of $\Omega^{n}\left(P_{0}, K_{0}\right)$ is virtually periodic.

Proof. Let $\left(P_{n}, K_{n}\right) \in \Omega^{n}\left(P_{0}, K_{0}\right)$. From the definition of $\Omega$ we see that there is a sequence of pairs $\left(P_{1}, K_{1}\right), \ldots,\left(P_{n-1}, K_{n-1}\right)$ so that $\left(P_{i+1}, K_{i+1}\right)$ belongs to $\Omega\left(P_{i}, K_{i}\right)$ for all $i=0,1, \ldots, n-1$. Since there are $\operatorname{dim}_{k} \operatorname{rad} \Lambda$ distinct isomorphism classes of syzygy pairs (1.1), the same pair must occur twice in the sequence. That pair is periodic, so the last pair $\left(P_{n}, K_{n}\right)$ must be virtually periodic.

We are ready to prove our main result.

Proof of Theorem A. Suppose $M$ has finite injective dimension. An immediate application of Lemmas 1.6 and 1.7 shows that $\operatorname{Ext}_{\Lambda}^{n}(K, M)=0$ for any syzygy pair $(P, K)$ and $n \geq \operatorname{dim}_{k}$ rad $\Lambda$. But the radical of $P_{v}$ is the direct sum of all $K_{\gamma}$ where $\gamma$ runs over all the arrows (paths of length 1) starting at $v$. Thus $\operatorname{Ext}_{\Lambda}^{n+1}\left(S_{v}, M\right)=0$ for every simple module $S_{v}$ and

$$
\text { inj } \operatorname{dim}_{\Lambda} M \leq \operatorname{dim}_{k} \operatorname{rad} \Lambda \text {. }
$$

Another immediate application of our results is the following:

Proposition 1.8. A monomial algebra has infinite global dimension if and only if there is a periodic syzygy pair.

\section{REFERENCES}

[GHZ] E. L. Green, D. Happel and D. Zacharia, Projective resolutions over Artin algebras with zero relations, Illinois J. Math. 29 (1985), 180-190.

[GKK] E. L. Green, E. Kirkman and J. Kuzmanovich, Finitistic dimensions of finite dimensional monomial algebras, (preprint), November, 1988.

Department of Mathematics, Brandeis University, Waltham, Massachusetts 02254

Department of Mathematics, Syracuse University, Syracuse, New York 13244 